BACTERIAL VAGINOSIS

\title{
Bacterial vaginosis in relation to menstrual cycle, menstrual protection method, and sexual intercourse in rural Gambian women
}

\author{
L Morison, G Ekpo, B West, E Demba, P Mayaud, R Coleman, R Bailey, G Walraven
}

Sex Transm Infect 2005;81:242-247. doi: 10.1136/sti.2004.011684

See end of article for authors' affiliations .....................

Correspondence to: Linda Morison, MRC Tropical Epidemiology Group, London School of Hygiene and Tropical Medicine, London WC1E 7HT, UK; linda.morison@ Ishtm.ac.uk

Accepted for publication 29 September 2004

\begin{abstract}
Objective: To examine the occurrence of bacterial vaginosis (BV) over the menstrual cycle and in relation to menstrual protection materials and sexual intercourse in a rural African setting.

Methods: Married, regularly menstruating female volunteers were asked to collect self administered swabs on alternate days through four menstrual cycles. BV was assessed using Nugent scores. Menstruation and reported sexual intercourse data were recorded contemporaneously. A crossover design comparing traditional and modern menstrual protection methods was incorporated. Multivariate logistic regression was used to examine associations with BV.

Results: 30 women completed four menstrual cycles in the study. Completeness and validity of data from the self administered swabs was high. Greater frequencies of BV were found for all women in the second week of the menstrual cycle relative to days 14t, and markedly higher frequencies of BV were found in the first week in women with infrequent $B V$. BV was (non-significantly) more frequent when modern pads were used compared with traditional cloths. No association was found between BV and intercourse reported in the previous 4 days; or between the frequency of reported intercourse in one menstrual cycle and BV in either the same menstrual cycle or the next.

Conclusions: Similar transient fluctuations over the menstrual cycle were found to those in industrialised countries. We found no evidence that sexual intercourse was associated with increased frequency of BV. Our data do not support hypotheses that menstrual hygiene materials might explain the high prevalences of BV found in sub-Saharan Africa compared to industrialised countries.
\end{abstract}

METHODS

Volunteers for the study were sought from five villages that had previously been included in a reproductive morbidity survey. ${ }^{8}$ We aimed to obtain a sample size at least as large as similar previously published longitudinal studies, ${ }^{45}$ which included 22 and 14 volunteers respectively. We therefore aimed to enrol around 40 women with the expectation of obtaining complete data for around 30 . Women were eligible for recruitment if they were asymptomatic at the previous survey, were married, did not intend to travel out of the study area for the duration of the study, and reported using no contraception and having had regular periods for the previous 3 months. Verbal consent was sought from husbands and signed consent was obtained from women. Each woman was examined by a gynaecologist to exclude local vaginal or cervical lesions including acute vaginitis, cervicitis, or polyps; and vaginal and cervical swabs were taken to screen for sexually transmitted infections, candidosis, and $\mathrm{BV}$. The laboratory methods used were as described for the previous survey. ${ }^{8}$ Women with any abnormalities or any infections other than BV were treated and excluded from study.

Women in this rural area of the Gambia use old cloths tied to the waist, washed, and re-used as required, for menstrual hygiene protection. A crossover comparison of menstrual hygiene protection methods was incorporated into the study whereby half the women used modern sanitary pads for the first two cycles and the other half used them in the second two cycles. Using this design the frequency of BV using the two methods of sanitary protection could be compared using each woman as her own control. Two packs of 10 sanitary pads with pants were supplied for each of the two cycles a woman was designated to use them, after which the remaining pads were retrieved. Women were given the pads

Abbreviations: $B V$, bacterial vaginosis 
at least 3 days before onset of menstruation. After the women completed two cycles using each method of sanitary protection they were asked how often they changed the sanitary protection at peak flow and which method they preferred. At the end of the study all participating women were given a 2 month supply of sanitary pads.

Women were taught how to take a high vaginal swab and given a supply of cotton tipped swabs in a sterile container. They were asked to take a swab first thing in the morning as soon as they got out of bed, on every other day. Swabs were taken on the days leading up to menstruation for the first cycle to accustom women to the technique and so as not to miss the beginning of the first cycle. Similarly, several swabs were taken in the fifth cycle so that the end of the fourth cycle could be determined accurately. Data collection took place between July and December 2000.

Swabs were collected before 9 am on the morning taken. On collection a brief questionnaire was administered on vaginal discharge, itching, irritation or bad odour in the genital area, sexual intercourse during the previous night or the night before, the last time they bathed before taking the swab, whether menstruating, and if menstruating the method of sanitary protection used. The woman's age, marital status, and circumcision status were obtained from data collected in the previous survey. ${ }^{8}$

The study was approved by the ethics committee of the Gambia Government Medical Research Council Laboratories (SCC proposal 832).

\section{Laboratory methods}

The swabs were stored in a cool box during transportation to the laboratory. Shortly after arrival (and within 2 hours of being taken) they were scored macroscopically for blood and rolled over a glass slide to make a smear which was then heat fixed. Slides were later scored for BV by Nugent's criteria. ${ }^{17}$ Where the Nugent scoring was based on scanty material this was noted, and slides with insufficient material were not read. A small validation study showed that this procedure resulted in identical Nugent scores to swabs rolled at the bedside. For all Nugent scoring the laboratory technician was blinded to any of the other data collected from the woman. Quality control of the readings was checked by rereading $10 \%$ of the slides by a second experienced reader. Vaginal swabs collected by the gynaecologist at initial recruitment and subsequent examinations were processed in parallel.

\section{Statistical methods}

Data were double entered into Epi-info v6.4 (CDC, Atlanta, GA, USA) and analysed using Stata version 7 (Statacorp, TX, USA). Graphs showing Nugent score over time for each woman used a moving average of Nugent scores to remove some of the noise in the graphs and make the patterns over time clearer. The moving average in this case was the average of the score at that time point and the score immediately before and after that time point. BV was defined as a Nugent score of 7-10, while scores of 4-6 and 0-3 were labelled "intermediate flora" and "normal flora," respectively. In order to identify which women had changes in the frequency of BV between cycles, the proportion of readings positive for BV was compared between cycles using Fisher's exact test. A non-parametric test (Kruskal-Wallis) was used to compare actual scores between cycles. Women were then grouped into four categories (BV group) according to their overall frequency of positive readings and whether change had occurred across the four cycles.

The association between stage of menstrual cycle, reported recent sex, and BV was examined using logistic regression models. Normal and intermediate flora categories (Nugent score 0-6) were combined to form the category "absence of
Table 1 Characteristics of the 30 women included in the analysis

\begin{tabular}{lc}
\hline Characteristic & $\begin{array}{c}\text { Number (\%) of } \\
\text { women }\end{array}$ \\
\hline $\begin{array}{l}\text { Ethnic group } \\
\text { Mandinka }\end{array}$ & $14(47)$ \\
Wolof & $9(30)$ \\
Fula & $7(23)$ \\
Number of co-wives* & \\
0 & $6(21)$ \\
1 & $20(69)$ \\
2 & $1(3)$ \\
$3-5$ & $2(7)$ \\
Age (years) & $10(33)$ \\
$20-30$ & $13(43)$ \\
$31-40$ & $7(23)$ \\
$41-53$ & $14(48)$ \\
Circumcision status* & $15(52)$ \\
Uncircumcised & \\
Circumcised & $2(7)$ \\
$\%$ nights on which sex with spouse was reported \\
0 & $5(17)$ \\
$0.1-10$ & $11(37)$ \\
$10.1-20$ & $5(17)$ \\
$20.1-30$ & $5(17)$ \\
$30.1-40$ & $2(7)$ \\
$40.1-50$ & \\
\hline &
\end{tabular}

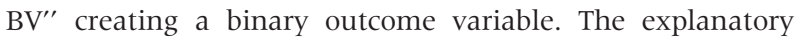
variables included in the models were stage of menstrual cycle measured in days since the start of the cycle $(<7$ days, 7-13 days, 14+ days) and whether the woman reported sex within the past 4 days. A variable indicating "BV group" was included in the models and interactions of the explanatory variables with BV group were examined. The type of sanitary protection used at the beginning of the current cycle was included as a possible confounder. Robust standard errors were calculated to adjust for likely correlation between BV readings for any particular woman.

Analysis of variance was used to compare the proportion of $\mathrm{BV}$ positive readings between the two sanitary protection methods within each woman (that is, including woman as a factor in the model) adjusting for crossover period. Only the data from cycles two and four was used (that is, considering months 1 and 3 as "washout" periods). Before analysis data points were log transformed so that differences on the scale of analysis would correspond to ratios of the original data. Where a woman had no readings positive for BV the number of $\mathrm{BV}$ positives was set to $0.5^{18}$ to avoid the data being set to missing after log transformation. Following the analysis of variance a non-parametric (matched pairs Wilcoxon) test was performed to assess the robustness of the results. An

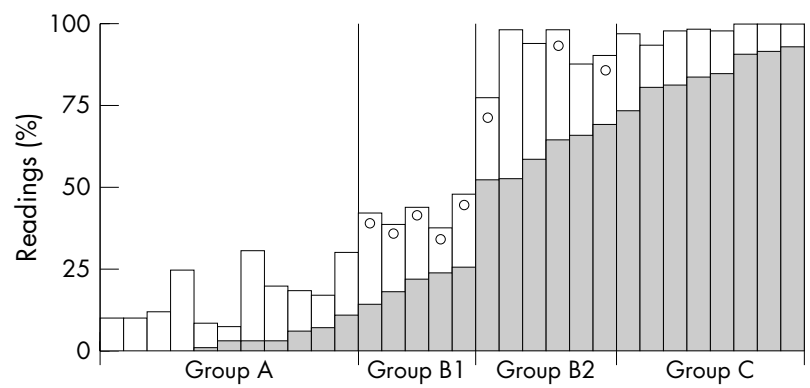

Figure 1 For each woman, the proportion of readings with $B V$ and intermediate flora; whether there were changes over the four cycles; and the "BV group" into which she was categorised. Shaded bars, BV (Nugent score 7-10); open bars, intermediate BV (Nugent score 4-6); open circles, significant change in BV over four cycles. 

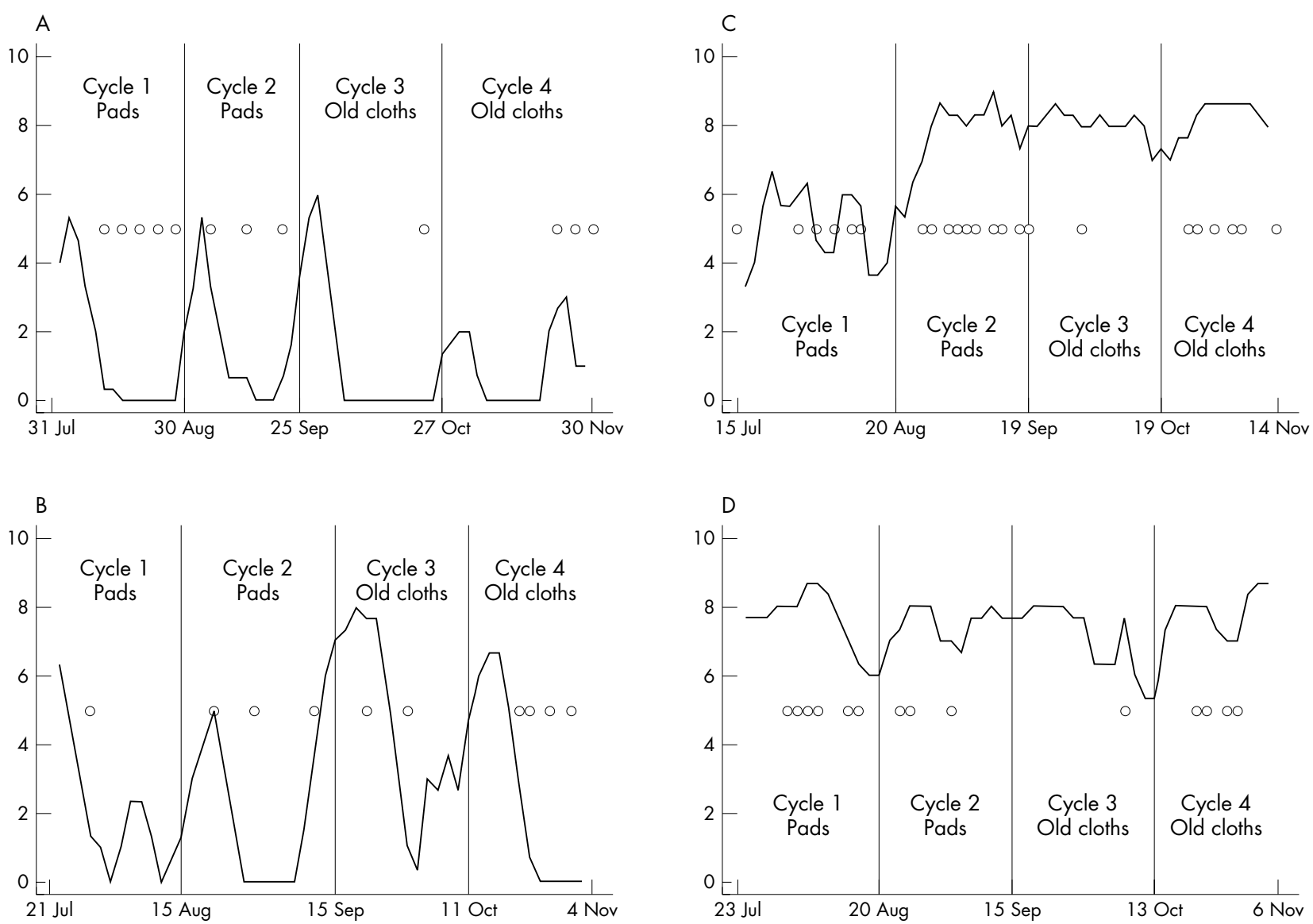

Figure 2 Smoothed Nugent scores over four menstrual cycles plotted for one woman from each BV group. (A) BV group A: stable, low frequency of BV. (B) BV group B1: changes over time and overall a low medium frequency of BV. (C) BV group B2: changes over time and overall a medium high frequency of BV. (D) BV group C: stable, high frequency of BV. Solid lines, Nugent score; circles, reported sexual intercourse during either/both of the previous two nights.

adjusted analysis was then conducted using a measure of BV standardised for reported recent sex and stage of menstrual cycle. A logistic regression model was fitted to each BV reading containing all explanatory variables except sanitary protection method. Adjusted measures were obtained by dividing the sum of the predicted number of $\mathrm{BV}$ positive readings by the sum of the observed number of $\mathrm{BV}$ positive readings (setting zeros to 0.5 as above) for each sanitary protection method for each woman. The standardised ratios were then analysed in the same way as for the unadjusted analysis. This method of obtaining and analysing standardised measures has been used previously for pair matched community randomised trials. ${ }^{19}$

\section{RESULTS}

\section{Samples obtained}

Forty one eligible women consented to take part in the study. One subsequently dropped out after providing no swabs, another four dropped out after providing between seven and nine swabs, and another dropped out after providing 24 swabs. The remaining 35 women provided between 53 and 84 swabs each. Five of these women were excluded from the analysis as they did not have four menstrual cycles over the study period-three because they became pregnant and the other two because they apparently had only two very long cycles. Another woman became pregnant during the fourth menstrual cycle so her data for the fourth cycle were truncated at 28 days. Table 1 summarises the characteristics of the 30 volunteers included in the analysis. Polygamy and female circumcision were common. No women had symptoms that caused them to seek clinical help.

For each woman the data for cycle zero (that is, before the first menstruation in the study period) and cycle five were discarded before statistical analysis. A total of 1724 swabs were provided by the 30 women over four menstrual cycles. No Nugent score was assigned to $28(1.6 \%)$ of the swabs owing to lack of material on the slide. The remaining 1696 were assigned a Nugent score, although 26 (1.5\%) of these were based on scanty material.

For 173 of the self administered swabs, Nugent scores from a swab taken by a gynaecologist on the same day were also available; 133 (76.9\%) agreed exactly and $162(93.6 \%)$ agreed within a score of 1 . For only eight $(4.6 \%)$ of the readings would discrepancies have altered the BV diagnosis.

The number of days over which the 30 women had four menstrual cycles varied from 90 to 156. For 21 women cycle length was always in the range 21-35 days. The other nine women had at least one cycle that fell out of this range (minimum 16 days, maximum 56 days). Five women had a complete set of readable swabs and 14 had missing scores on less than $5 \%$ of days. Four women had between $5 \%-9 \%$ of scores missing and six had 10\%-14\% missing. One woman had $29 \%$ of her readings missing mainly because she went away twice, each time for over a week.

\section{Descriptive analysis of Nugent scores}

Figure 1 shows how the frequency of BV positive readings and changes over time were used to divide the women into 


\begin{tabular}{|c|c|c|c|c|}
\hline Stage of menstrual cycle & $\begin{array}{l}\text { BV positive/ } \\
\text { all readings }\end{array}$ & $\%$ & $\mathrm{OR}^{*}$ & $95 \% \mathrm{Cl}$ \\
\hline \multicolumn{5}{|l|}{ BV group $A(n=11) \dagger$} \\
\hline $14+$ days & $1 / 350$ & 0.3 & 1 & \\
\hline$<7$ days & $15 / 165$ & 9.1 & 35.9 & 4.8 to 268.6 \\
\hline $7-13$ days & $5 / 123$ & 4.1 & 14.9 & 1.4 to 152.2 \\
\hline \multicolumn{5}{|l|}{ BV group B1 $(n=5)$} \\
\hline $14+$ days & $15 / 143$ & 10.5 & 1 & \\
\hline$<7$ days & $29 / 77$ & 37.7 & 6.56 & 4.39 to 9.80 \\
\hline 7-13 days & $14 / 58$ & 24.1 & 2.51 & 1.29 to 4.89 \\
\hline \multicolumn{5}{|l|}{ BV group B2 $(n=6)$} \\
\hline $14+$ days & $93 / 163$ & 57.1 & 1 & \\
\hline$<7$ days & $57 / 94$ & 60.6 & 1.35 & 0.58 to 3.18 \\
\hline 7-13 days & $51 / 70$ & 72.9 & 2.35 & 1.74 to 3.18 \\
\hline \multicolumn{5}{|l|}{ BV group C ( $n=8)$} \\
\hline $14+$ days & $201 / 239$ & 84.1 & 1 & \\
\hline$<7$ days & $100 / 124$ & 80.6 & 0.81 & 0.42 to 1.54 \\
\hline 7-13 days & $84 / 90$ & 93.3 & 2.60 & 1.24 to 5.44 \\
\hline
\end{tabular}

four BV groups. Group A had BV on less than $15 \%$ of readings and no significant differences between cycles. Groups Bl and B2 showed some significant differences between cycles with between $16 \%$ and $30 \%$ of readings BV positive for group Bl and between $50 \%$ and $70 \%$ of readings BV positive for group B2. Group C were BV positive on more than $70 \%$ of readings and had no significant differences between cycles. In summary, group A represents those with a stable low frequency of $\mathrm{BV}$ and group $\mathrm{C}$ those with a stable high frequency of BV. Group B represents those whose BV pattern changed with Bl generally having a medium to low frequency and B2 a medium to high frequency.

To illustrate the types of pattern seen, figure 2 shows smoothed Nugent scores plotted over time for one woman from each of the four BV groups. A pattern for increased Nugent scores at the beginning of each menstrual cycle is apparent for the women with lower frequencies of positive $\mathrm{BV}$ readings (that is, groups $\mathrm{A}$ and $\mathrm{Bl}$ shown in fig $2 \mathrm{~A}$ and $2 \mathrm{~B}$, respectively).

\section{BV and stage of menstrual cycle}

The association between BV and stage of menstrual cycle differed by BV group $(p<0.05$ for interaction between stage of menstrual cycle and BV group). Table 2 shows that in those with low and stable BV (group A) there was very little BV in days 14+ of the cycle, with a markedly higher odds of $\mathrm{BV}$ in the first 6 days and a higher odds also in days 7-14 (although confidence intervals were wide). A similar but less marked pattern could be seen for group B1. In groups B2 and $\mathrm{C}$ the odds of a positive $\mathrm{BV}$ reading were not significantly higher for the first 6 days of the cycle relative to days 14+ but were significantly higher for days 7-14.

\section{BV and reported sexual intercourse}

BV arose on 126 occasions over the study period when data on reported sexual intercourse were also available; 59 of these $(46.8 \%)$ were within 4 days of reported sex. A similar proportion of BV episodes regressed within 4 days of reported sex $(65 / 127 ; 51.2 \%)$. The logistic regression models showed little association between reported sex in the past 4 days and $\mathrm{BV}$. The adjusted odds ratio for a positive BV reading when sex was reported in the last 4 days relative to no reported sex in the past 4 days was 0.98 (95\% CI: 0.66 to 1.46). No significant interaction with BV group was found.

A further analysis was conducted to examine whether the frequency of sex reported in one menstrual cycle was associated with the frequency of BV in the next. Regression analysis was conducted on data aggregated by woman and cycle, using robust standard errors to take account of correlation within woman. No evidence of association $(p=0.760)$ was found. There was also no association between frequency of sex and frequency of BV in the same month $(p=0.770)$. When the change in BV frequency from the previous month to the present one was regressed against the frequency of sex in the previous month again no association was found $(\mathrm{p}=0.582)$.

\section{Comparison of sanitary protection methods}

For the comparison of sanitary protection methods one of the 30 women had to be excluded owing to an error in the distribution of sanitary pads. The adjusted and unadjusted ratios for the risk of $\mathrm{BV}$ using pads relative to traditional cloths were identical at $1.44(\mathrm{p}=0.083 ; 95 \%$ CI 0.95 to 2.18 for the crude analysis; $p=0.066$; $95 \%$ CI: 0.97 to 2.12 for the adjusted analysis) suggesting that if anything a higher frequency of BV was found when sanitary pads were used. The matched pairs Wilcoxon test gave a p value of 0.330 .

The interviews on preference of type of sanitary protection included 27 of the 30 study women and all but one said they preferred the pads (the other woman expressed no preference). No women reported that they had washed and reused a pad. The range of response to questions about frequency of changing the pad in 1 day at peak flow varied more for the traditional cloths (1-6) than the pads (2-5) but the average was not significantly different (3.1 for cloths and 2.9 for pads $\mathrm{p}=0.557$ from paired $t$ test, $\mathrm{p}=0.495$ from matched pairs Wilcoxon test).

\section{DISCUSSION}

To our knowledge this is the first longitudinal study of BV in a rural African setting. In the study area BV prevalence is high $\left(37 \%^{8}\right)$, polygamy is common, and traditional methods of sanitary protection are used. The 30 women in this study were married, free of STI, and not using any modern contraceptive methods. We obtained a high degree of completeness of swab collection despite logistical difficulties. Similar to previous studies ${ }^{20}{ }^{21}$ we also found a high level of validity of readings from the self administered swabs even though the swabs in this study were not rolled immediately onto slides There was large variation in the length of the menstrual cycles of the women in this study but this was consistent with studies on menstrual cycle length from industrialised countries. ${ }^{22}$

Similar to studies conducted in industrialised countries ${ }^{1-7}$ we found a high degree of fluctuation in women's patterns of vaginal flora over time. Only four women had Nugent scores 
less than 7 for all four menstrual cycles and even they had intermediate flora some of the time. A pattern of highest BV frequency in the first week of the menstrual cycle, with spontaneous resolution occurring after day 14, was apparent only in women with a relatively low frequency of BV. This is consistent with findings from industrialised countries of women with relatively infrequent $\mathrm{BV} .{ }^{5-7}$ It is still not clear whether changes in the vaginal flora in the first week of menstruation are brought about by the presence of menstrual blood and/or hormonally. A tendency for BV to resolve in pregnancy ${ }^{23-25}$ could be because of lack of menstruation and/ or hormonal effects. Lower prevalences of BV in women on hormonal contraceptives ${ }^{26}$ and hormonally induced changes in vaginal flora in mice ${ }^{28}$ perhaps favour the role of hormones but the mechanisms of any such influence are not understood.

Given the established cyclic fluctuations in vaginal flora the question is what "tips the balance" so that more persistent patterns of BV are seen. Sexual intercourse has been hypothesised as a possible factor through alterations to the vaginal $\mathrm{pH},{ }^{5}$ the direct introduction into the vagina of $\mathrm{BV}$ related organisms, ${ }^{29}$ or lactobacilli destroying phages. ${ }^{30}$ In this setting there was no evidence of a short term (within 4 days) association between reported sexual intercourse and BV, in contrast with the findings of a previous study which found spontaneous resolution to be associated with recent intercourse. ${ }^{5}$ We also did not find any association between frequency of intercourse and BV in the following menstrual cycle. However, a possible limitation of the study is that we relied on women's reports of when sexual intercourse took place. Recall bias is unlikely, as women were asked about this every other day, but the reports might be subject to some social desirability bias.

Studies in industrialised countries have found higher prevalences of $\mathrm{BV}$ in women with a higher rate of partner change. ${ }^{262731}$ In addition to possible extramarital relationships, a substantial proportion of marriages in the study area are polygamous so the husband would have concurrent sexual partners. BV concordance within marriage sets and the effect of the types of partner changes associated with polygamy merit further research.

Our study does not provide any evidence that differences in menstrual hygiene practice explain the differences in prevalence of BV seen between sub-Saharan Africa and industrialised countries. However, this study only included 30 volunteers and each method was used for only two cycles in the study. Our data could be used to inform frequency of measurement and sample sizes necessary for a full scale randomised trial of menstrual hygiene methods in developing countries.

Given the poor understanding of the role of hormones in the aetiology of BV more research is needed in this area. ${ }^{31}$ One approach would be to relate reproductive hormone levels

\section{Key messages}

- Consistent with studies in industrialised countries this study found higher prevalences of BV in the first week of the menstrual cycle in women with relatively infrequent BV

- No association was found between BV and reporting sex in the previous 4 days or the frequency of sex reported in the previous menstrual cycle

- The use of modern rather than traditional menstrual protection methods for two menstrual cycles did not result in a reduced frequency of BV to vaginal flora longitudinally over the menstrual cycle. Hormones and vaginal flora patterns in women could also be compared before and after hormonal intervention (such as oral contraception). A third approach would be to examine levels of hormones and BV in women of African and nonAfrican origin both in Africa and in industrialised countries. It is possible that hormonal differences explain some of the disparities in prevalence of BV seen between Afro-Caribbean and white women within industrialised countries, and between women in sub-Saharan Africa and industrialised countries. However, such studies are complicated by factors such as diet and body mass index (for example, malnutrition or obesity) which vary between racial groups and settings and affect both hormones and disease, as has been found in research on breast cancer in the United States. ${ }^{32}{ }^{33}$

\section{CONCLUSIONS}

Our data showed similar transient fluctuations over the menstrual cycle as previous studies in industrialised settings in women with relatively infrequent BV. Modern methods of menstrual hygiene protection used for two menstrual cycles were not associated with a lower frequency of BV relative to traditional methods, supplying no evidence for the hypothesis that traditional methods of menstrual hygiene could explain the high prevalences of BV observed in sub-Saharan Africa. In this setting where polygamous marriages were common, we observed no association between reported sexual intercourse and BV either over the short term (within 4 days) or between menstrual cycles. The roles of reproductive hormones and sexual behaviour patterns in the aetiology of BV merit further research.

\section{ACKNOWLEDGEMENTS}

We thank the field workers, led by Hawa Manneh, Emily Loppy, and Hadijatou Jallow, for their help in collecting the data; Nkaye Kanyi for laboratory help; and Maimuna Sowe for help with data processing. We also thank Basia Zaba and Maarten Schim for helpful discussions regarding the study.

\section{CONTRIBUTORS}

LM analysed the data and wrote the main body of the paper; GW and PM designed the study; GW, GE, and RC supervised the data collection; and BW and ED supervised and conducted laboratory analysis; all co-authors commented on drafts of the paper.

\section{Authors' affiliations}

L Morison, P Mayaud, R Bailey, Department of Infectious and Tropical Diseases, London School of Hygiene and Tropical Medicine, London, UK G Ekpo, B West, E Demba, R Coleman, R Bailey, G Walraven, Medical Research Council (MRC) Laboratories, Fajara, the Gambia

Funding: This study was funded by the British Medical Research Council. Conflict of interest: none.

\section{REFERENCES}

1 Bartlett JG, Onderdonk AB, Drude E, et al. Quantitative bacteriology of the vaginal flora. J Infect Dis 1977;136:271-7.

2 Sautter RL, Brown WJ. Sequential vaginal cultures from normal young women. J Clin Microbiol 1980;1 1:479-84.

3 Onderdonk AB, Zamarchi GR, Walsh JA, et al. Methods for quantitative and qualitative evaluation of vaginal microflora during menstruation. Appl Environ Microbiol 1986;51:333-9.

4 Keane FEA, Ison CA, Taylor Robinson D. A longitudinal study of the vaginal flora over a menstrual cycle. Int J STD AIDS 1997;8:489-94.

5 Hay PE, Ugwumadu A, Chowns J. Sex, thrush and bacterial vaginosis. Int J STD AIDS 1997;8:603-8.

6 Schwebke JR, Richey CM, Weiss HL. Correlation of behaviours with microbiological changes in vaginal flora. J Infect Dis 1999;180:1632-6.

7 Eschenbach DA, Thwin SS, Patton DL, et al. Influence of the normal menstrual cycle on vaginal tissue, discharge, and microflora. Clin Infect Dis 2000;30:901-7.

8 Walraven G, Scherf C, West B, et al. The burden of reproductive-organ disease in rural women in the Gambia, West Africa. Lancet 2001;357:1161-7. 
9 Sewankambo N, Gray RH, Wawer MJ, et al. HIV-1 infection associated with abnormal vaginal flora morphology and bacterial vaginosis. Lancet 1997;350:546-50.

10 Govender L, Hoosen AA, Moodley J, et al. Bacterial vaginosis and associated infections in pregnancy. Int J Gynaecol Obstet 1996;55:23-8.

11 Mayaud P, Uledi E, Cornelissen J, et al. Risk scores to detect cervical infections in urban antenatal clinic attenders in Mwanza, Tanzania. Sex Transm Infect 1998;74(Supp 1):S139-46.

12 Cristiano L, Rampello S, Noris C, et al. Bacterial vaginosis: prevalence in an Italian population of asymptomatic pregnant women and diagnostic aspects. Eur J Epidem 1996;12:383-90.

13 Delaney ML, Onderdonk AB. Nugent score related to vaginal culture in pregnant women. Obstet Gynecol 2001;98:79-84.

14 Wasserheit JN, Harris JR, Chakraborty J, et al. Reproductive tract infections in a family planning population in rural Bangladesh. Stud Family Planning 1989;20:69-80.

15 Younis N, Khattab H, Zurayk H, et al. A community study of gynecological and related morbidities in rural Egypt. Stud Family Planning 1993;24:175-86

16 Bulut A, Filippi V, Marshall T, et al. Contraceptive choices and reproductive morbidity in Istanbul. Stud in Family Planning 1997;28:35-43.

17 Nugent RP, Krohn MA, Hillier SL. Reliability of diagnosing bacterial vaginosis is improved by a standardised methed of gram stain interpretation. J Clin Microbiol 1991;29:297-301.

18 Armitage P, Berry P. Statistical methods in medical research. Oxford: Blackwell, 1994.

19 Grosskurth H, Mosha F, Todd J, et al. Impact of improved treatment of sexually transmitted diseases on HIV infection in rural Tanzania: randomised controlled trial. Lancet 1995;346:530-6.

20 Schwebke JR, Morgan SC, Weiss HL. The use of sequential sself-obtained swabs for detecting changes in the vaginal flora. Sex Transm Dis 1997;24:236-9.

21 Boskey ER, Atherly-Trim SA, O'Campo PJ, et al. Acceptability of a selfsampling technique to collect vaginal smears for gram stain diagnosis of bacterial vaginosis. Women's Health Issues 2004;14:14-18.
22 Trelour AE, Boynton RE, Behn BG, et al. Variation of the human menstrual cycle through reproductive life. Int J Fertil 1967;13:77-126.

23 Hay PE, Morgan DJ, Ison CA, et al. A longitudinal study of bacterial vaginosis during pregnancy. Br J Obstet Gynaecol 1994;101:1048-53.

24 Klebanoff MA, Hauth JC, MacPherson CA, et al. for National Institute for Child Health and Development Maternal Fetal Medicine Units Network. Time course of the regression of asymptomatic bacterial vaginosis in pregnancy with and without treatment. Am J Obstet Gynecol 2004; 190:363-70

25 Ugwumadu A, Reid F, Hay P, et al. Natural history of bacterial vaginosis and intermediate flora in pregnancy and effect of oral clindamycin. Obstet Gynecol 2004;104:114-19.

26 Smart S, Singal A, Mindel A. Social and sexual risk factors for bacterial vaginosis. Sex Transm Infect 2004;80:58-62.

27 Holzman C, Leventhal JM, Qiu H, et al, and the BV Study Group. Factors linked to bacterial vaginosis in non-pregnant women. Am J Public Health 2001;91:1664-1670.

28 Sonnex C. Influence of overian hormones on urogenital infection. Sex Transm Infect 1998;74:11-19.

29 Keane FEA, Thomas BJ, Gilroy CB, et al. The association of Mycoplasma hominis, Ureaplasma urealyticum and Mycoplasma genitalium with bacterial vaginosis: observations on heterosexual women and their male partners. Int J STD AIDS 2000;11:356-60.

30 Kilic AO, Pavlova SI, Alpay S, et al. Comparative study of vaginal lactobacillus phages isolated from women in the United States and Turkey: prevalence, morphology, host range, and DNA homology. Clin Diag Lab Immun $2001 ; 8: 31-9$

31 Taylor-Robinson D. The future of bacterial vaginosis research. Int J Gynecol Obstet 1999:67:s35-8.

32 Haiman CA, Pike MC, Bernstein L, et al. Ethnic differences in ovulatory function in nulliparous women. Br J Cancer 2002;86:367-71.

33 Woods MN, Barnett JB, Spiegalman D, et al. Hormone levels during dietary changes in pre-menopausal African-American women. J Natl Cancer Inst 1996;88:1369-74. 